\title{
DESVELANDO A VIVÊNCIA DA EQUIPE MULTIPROFISSIONAL NO CUIDAR DO PACIENTE ESQUIZOFRÊNICO
}

\section{Patricia Suguyama}

Universidade Estadual de Maringá (UEM), Maringá (PR), Brasil

E-mail: patty_suguyama@hotmail.com

\section{Laís Stocco Buzzo}

Centro Universitário Cesumar (UniCesumar), Maringá (PR), Brasil.

Magda Lúcia Félix de Oliveira

Universidade Estadual de Maringá (UEM), Maringá (PR), Brasil.
RESUMO: A esquizofrenia é considerada um transtorno mental grave e crônico, que possui um rol de sintomas. Assim, é imprescindível compreender o contexto no qual se insere o paciente, para determinar quais intervenções contribuirão para o seu tratamento. Nesse sentido, o trabalho em equipe tem por fim a reorganização do serviço para promover qualidade de vida ao paciente. Objetivou-se, portanto, compreender a vivência da equipe multiprofissional na atenção ao paciente esquizofrênico, por meio de uma pesquisa descritivo-exploratória, com abordagem qualitativa. Participaram da pesquisa dez profissionais de um Hospital Psiquiátrico situado no Norte do Paraná. Os dados foram analisados conforme Bardin e, segundo a análise de conteúdo, foram identificadas duas categorias temáticas: Vivência da equipe multiprofissional com o paciente esquizofrênico e Importância da equipe multiprofissional na assistência ao paciente esquizofrênico. $\mathrm{O}$ resultado revelou que os entrevistados, além de trabalharem em equipe, reconheceram sua importância no tratamento do paciente e que a interdisciplinaridade faz parte da realidade dos mesmos, no qual mostram-se evidentes nas falas.

PALAVRAS-CHAVE: Equipe de Assistência ao Paciente; Esquizofrenia; Saúde Mental; Transtornos Mentais.

\section{THE EXPERIENCE OF THE MULTI-PROFESSIONAL TEAM IN THE CARE OF A SCHIZOPHRENIC PATIENT}

ABSTRACT: Schizophrenia is a serious and chronic mental disorder with a great number of symptoms. It is highly relevant to know in which context the patient is inserted to determine interventions for treatment. Team work aims at organizing improvement in the patient's life quality. The experience of the multi-professional team is important within attention to the schizophrenic patients through a descriptive, quality and exploratory research. Ten professionals of a Psychiatric Hospital in the northern region of the state of Paraná, Brazil, researchers participated. They analyzed data following Bardin's method and two thematic categories were identified by content analysis: Experience of the multi-professional team with the schizophrenic patient and importance of the multi-professional team in the assistance to the schizophrenic patient. Results revealed that the interviewed, working as a team, acknowledged their important in the patient's treatment and that interdisciplinarity is part and parcel to the world they live in, shown in their discourses.

KEY WORDS: Assistance Team to the Patient; Schizophrenia; Mental Health; Mental Disorder. 


\section{INTRODUÇÃO}

Dados epidemiológicos apontam a esquizofrenia como um problema mundial de saúde pública e que, em média, esses pacientes chegam a ocupar $50 \%$ dos leitos dos hospitais psiquiátricos, constituindo-se em um problema social e sanitário (KOGA; FUREGATO, 2002; RESENDE; VIGLIONE; ARGIMON, 2009; CORDEIRO et al., 2012).

Além disso, a esquizofrenia é considerada um dos transtornos mentais mais graves, de acordo com a Organização Mundial da Saúde (2001), e é a terceira causa de perda da qualidade de vida entre os 15 e 44 anos, o que impede que o indivíduo atinja o auge do seu potencial (KOGA; FUREGATO, 2002; CORDEIRO et al., 2012). Para os profissionais da área da saúde representa um grande desafio assistencial, pois seu curso leva à cronicidade dos sintomas (ALMEIDA et al., 2010).

Avanços científicos recentes trouxeram mudanças para o tratamento da doença. Os medicamentos disponibilizados controlam os delírios e alucinações, mas, além dos medicamentos, a participação da equipe multiprofissional viabiliza a reinserção social e a melhora nas condições de vida dos pacientes (NOTO; BRESSAN, 2012).

A equipe multiprofissional revela-se como uma estratégia para redesenhar a assistência e promover a qualidade de vida ao paciente. É importante relembrar que, assim como a saúde passou a ser garantida constitucionalmente como "direito de todos e dever do Estado", na Constituição Federal de 1988, torna-se imprescindível a modificação no pensar dos profissionais, para o desenvolvimento de trabalhadores autônomos, com saberes em trabalho em equipe e o comprometimento com os requisitos atuais (ABUHAB et al., 2005; CORDEIRO et al., 2012).

Neste contexto, fica nítida a importância de mudanças e atitudes em relação à saúde mental, no entanto, para que ocorram tais modificações, é essencial que os profissionais se conscientizem dessas novas ideias e, assim, possam executar uma assistência digna e de qualidade que venha ao encontro dos princípios do SUS (NOTO; BRESSAN, 2012).

Delimitou-se como objetivo deste estudo compreender a vivência da equipe multiprofissional na atenção ao paciente esquizofrênico.

\section{METODOLOGIA}

Trata-se de uma pesquisa exploratória descritiva, que percorre a trajetória metodológica qualitativa, realizada em um Hospital Psiquiátrico situado ao Norte do Paraná. Participaram do estudo dez profissionais, que atenderam aos seguintes critérios de inclusão: trabalhar com pacientes esquizofrênicos e compor a equipe multiprofissional do Hospital Psiquiátrico.

Para a coleta de dados, construiu-se um questionário semiestruturado composto por duas perguntas norteadoras: 1) Como você vivencia o trabalho da equipe multiprofissional com o paciente esquizofrênico? 2) Qual a importância do trabalho multiprofissional para você, na instituição em que trabalha? Como pode descrevê-lo?

A coleta de dados foi realizada no período de julho a agosto de 2013. As entrevistas foram transcritas na íntegra e submetidas a duas rodadas de leitura, das quais foram retiradas palavras para apreciação dos dados, a partir da técnica de análise de conteúdo, proposta por Bardin (2008), buscando outras realidades através das mensagens, a partir das quais se estabeleceram três pontos cronológicos: pré-análise; exploração do material; e tratamento dos resultados: a inferência e a interpretação.

Após a exploração do material, foram identificadas duas categorias temáticas que nortearam a discussão. A primeira nomeada como Vivência da equipe multiprofissional com o paciente esquizofrênico e, a segunda, de Importância da equipe multiprofissional na assistência ao paciente esquizofrênico

$\mathrm{O}$ anonimato dos participantes da pesquisa foi garantido, após a assinatura do Termo de Consentimento Livre e Esclarecido (TCLE), bem como obedecido todos os aspectos presentes na Resolução n 466/2012, do Conselho Nacional de Saúde (BRASIL, 2012). No sentido de preservar os nomes dos depoentes, optou-se por identificá-los pela palavra "Profissional" seguida de número ordinal conforme a ordem de realização das entrevistas.

O projeto de pesquisa foi submetido ao Comitê de Ética em Pesquisa Envolvendo Seres Humanos do Centro Universitário Cesumar (UniCesumar), sob o processo $\mathrm{n}^{\mathrm{O}} 363.745$. 


\section{RESULTADOS}

\subsection{VIVÊNCIA DA EQUIPE MULTIPROFISSIONAL COM PACIENTE ESQUIZOFRÊNICO}

Os entrevistados relataram as diversas concepções sobre o paciente esquizofrênico, discorrendo sobre as visões adquiridas na prática do dia a dia:

[...] é um paciente que vai chegar delirante e desorganizado, a higiene bem precária, é por conta dos delírios que ele ouve das vozes, que dizem muitas vezes para ele não tomar banho (PROFISSIONAL 1).

Paciente que começa a falar sozinho, tem delírios, alucinações e, às vezes, até briga com outro paciente [...] são pacientes que sempre precisam de cuidados até apresentar a melhoria deles (PROFISSIONAL 7).

A paciência com esse paciente é algo muito citado entre os entrevistados, como se apresenta a seguir:

[...] eu procuro vivenciar, com muita calma e paciência, pois é um paciente que tem todo um transtorno. Um problema que vem desde o passado. Então, não sabemos se tem uma família, uma alimentação adequada. Uma base familiar que saiba lidar com essa doença (PROFISSIONAL 3).

[...] a pessoa sofre. Eu vejo assim, entender o lado da doença, para poder trabalhar com essas pessoas, sempre com muita paciência (PROFISSIONAL 4).

[...] temos que ter bastante paciência, porque eles falam sozinhos, falam com as vozes, temos que ver aquilo e tentar entender o mundo deles (PROFISSIONAL 9).

[...] não são só os cuidados, tem que ter paciência para escutar, eles têm muitas manias, eles têm um jeito de viverem (PROFISSIONAL 10).

\subsection{IMPORTÂNCIA DA EQUIPE MULTIPROFISSIONAL NA ASSISTÊNCIA AO PACIENTE ESQUIZOFRÊNICO}

O conjunto de ideias, sobre o processo de trabalho em equipe, é mencionado pelos entrevistados nos seguintes comentários:
[...] é importante se trabalhar em equipe, principalmente, na área da psiquiatria, no qual cada profissional focará algo no paciente, mas, de certa forma, todos trabalharão com o todo que é o paciente. $\mathrm{Na}$ área da saúde, há muito essa visão tecnicista, que segmenta o paciente, cada um vai trabalhar com uma parte só, porém, quando se trabalha em equipe, conseguimos chegar no todo que é o paciente (PROFISSIONAL 1).

Eu não consigo trabalhar de forma isolada, dependo muito da avaliação da equipe multiprofissional. Eu atendo o paciente, avalio a parte médica, a parte psiquiátrica (PROFISSIONAL 4).

Tudo é um conjunto que se tem que andar para no final se chegar àquele objetivo. Todos são importantes e se falta alguma coisa no final vai faltar também, sendo então um conjunto (PROFISSIONAL 6).

[...] enfim, aqui precisamos trabalhar em equipe, unidos, porque sozinho você não consegue (PROFISSIONAL 7).

[...] eu acho fundamental, pois não dá para tratar nenhum tipo de patologia, principalmente o transtorno mental, se não for com uma equipe multiprofissional $[. .$.$] com todos$ os olhares em cima desse paciente, para podermos ver ele como um todo, para não ter mais essa visão compartimentada. Ele tem transtorno mental e só vou ver a cabecinha dele, ele precisa ter qualidade de vida, precisa ter uma atividade física, precisar ter outras coisas para que ele melhore (PROFISSIONAL 8).

Para os entrevistados existe um intercâmbio de conhecimentos entre as especialidades, que visa enriquecer suas práticas diárias, reduzindo dúvidas, melhorando diagnósticos, bem como a terapêutica adequada, visando sempre proporcionar qualidade de vida para o paciente.

[...] então eu preciso dessa relação para poder fazer esse planejamento e para poder ter esse manejo com o paciente. Assim, também acredito que os profissionais necessitam da minha visão para poder ter um manejo adequado do paciente (PROFISSIONAL 2). 
[...] juntos vamos discutir o que é melhor para o paciente, o que ele pode fazer e o que não pode e qual tratamento para ele (PROFISSIONAL 5).

As reuniões de equipe, realizadas semanalmente como rotina da Instituição, às quartas-feiras, configuraram-se como um meio enriquecedor tanto da troca de conhecimentos entre as várias especialidades envolvidas, como também contribuem para a evolução dos pacientes, como se pode comprovar a partir dos relatos:

[...] primeiramente, a equipe multiprofissional se reúne para discutir sobre os pacientes. Cada profissional tem uma diretriz voltada para a área de atuação que seguirá com o paciente, por exemplo, a educação física é voltada para a atividade física; a psicóloga, para conversa, cada profissional tem sua rotina (PROFISSIONAL 5).

[...] é isso, estabelecer laço, saber quais são as dificuldades que os pacientes estão enfrentando [...] trazer os problemas para as reuniões e tentar auxiliar da melhor maneira em reuniões e isso a gente tentar resolver (PROFISSIONAL 2).

[...] equipe multiprofissional é a base do Hospital, porque, muitas vezes, eu não sei como lidar com determinada situação, então, peço ajuda para os outros profissionais. Então, sempre nos encontramos no Hospital uma vez por semana, de quarta-feira, e debatemos muito sobre isso (PROFISSIONAL 3).

A comunicação é fundamental, principalmente no trabalho em equipe. A interação entre os profissionais e as ações, assim como a associação de conhecimentos, flui, simbolicamente, pela linguagem e é por meio dela que os membros da equipe trocam informações, questionamentos, estabelecem consenso e delimitam planos de cuidados em comum acordo. Essa ideia é reforçada nos seguintes depoimentos:

[...] o mais importante é a comunicação, é ter uma inter-relação com os outros profissionais (PROFISSIONAL 9).
[...] é importante que todos tenham o mesmo objetivo em comum que é o paciente, tem que se falar uma linguagem que todos tenham acesso, ter esse diálogo, entre os profissionais (PROFISSIONAL 1).

A seguir, os entrevistados comentam sobre a importância da equipe na organização nos processos de trabalho com os pacientes:

[...] a importância do trabalho, ela é assim, cada um faz a sua parte, porém, um sempre acaba completando a parte do outro. Os pacientes acabam tendo muito tempo vago no Hospital, então torna-se necessário ter atividades para estar completando o horário que eles têm livre, como exemplo a Terapia Ocupacional, os professores de atividade física e a nutricionista. Um acaba encaixando o serviço de outro, é um corpo (PROFISSIONAL 10).

[...] se não houver a equipe, como um profissional só irá conseguir dar conta de vários setores? Por isso, é importante a equipe. Cada um ser responsável pelo seu setor e, depois, discutir, verificando se o paciente está evoluindo, onde estão os problemas, onde se está errando, se está acertando, se está no caminho certo. Por isso, a equipe é importante, cada um tem seu setor, mas trabalhando junto, para o bem do paciente (PROFISSIONAL 5).

[...] trabalhos em equipe, por exemplo, tem a psicóloga, a terapia ocupacional, que ajudam bastante na melhoria do paciente. Tem a nutrição que cuida da parte da alimentação e cada dia tem uma dieta diferente. Fazemos o máximo para trabalhar em equipe (PROFISSIONAL 7).

\section{DISCUSSÃO}

Para chegar à categoria Vivência da equipe multiprofissional com paciente esquizofrênico, encontrou-se um conjunto de significados que interferem no desenvolvimento do papel, do comportamento e das ações dos profissionais dentro da equipe multiprofissional na assistência ao paciente esquizofrênico. 
A esquizofrenia é um transtorno mental grave e crônico, no qual se apresenta repercussão em nível afetivo e cognitivo que acabam afetando nas relações interpessoais do indivíduo, limitando o paciente em seu funcionamento intelectual e em suas relações e comportamentos sociais (LAUTER et al., 2011; CORDEIRO et al., 2012).

Para os profissionais entrevistados, os pacientes esquizofrênicos, em sua maioria, apresentam-se desorganizados, agitados e a alucinação encontra-se muito presente, o que acaba gerando ansiedade, por parte desses profissionais. Esses sentimentos podem acabar dificultando o estabelecimento da comunicação interpessoal e a concretização da relação de ajuda, entre profissional e paciente (LAUTER et al., 2011; LIMA; GARCIA; TOLEDO, 2013).

A equipe multiprofissional deve entender a realidade que a doença impõe ao paciente, deixando-o vulnerável às circunstâncias de seu ambiente, podendo comprometer sua autonomia, afetando, direta ou indiretamente, várias etapas de sua vida (FERNANDES; SAEKI, 2009; CORDEIRO et al., 2012). Assim, a atitude terapêutica não é produto de mero instinto, mas são reações comportamentais que uma vez formadas por um profissional devem ser incorporadas ao seu modo de ação.

Sabendo que a esquizofrenia possui um rol de sintomatologias e seu curso acaba variando de acordo com cada indivíduo, torna-se essencial compreender o contexto pelo qual se insere esse paciente para determinar quais intervenções contribuirão no tratamento. Dessa maneira os profissionais entrevistados procuram entender o contexto deste paciente, para assim planejar as ações, no intuito de organizá-los em rotinas, para que sigam um processo sistematizado.

A abordagem realizada pelos entrevistados compreende um conjunto de ações capazes de proporcionar aos pacientes uma melhor integração social e, consequentemente, melhor qualidade de vida, considerando os limites impostos pelo transtorno mental, procurando visar às necessidades humanas básicas, bem como facilitando a comunicação, tanto entre os próprios profissionais como com o paciente.

Na categoria Importância da equipe multiprofissional na assistência ao paciente esquizofrênico, procu- rou-se ressaltar o trabalho em equipe multiprofissional realizado pelos entrevistados como prática norteadora dos serviços.

A internação psiquiátrica representa um momento de intenso sofrimento, tanto para o paciente, como para seus familiares, no qual a condição de isolar socialmente esse indivíduo sobre o abrigo do Hospital ocorre quando não há mais condições mínimas de segurança, que garantam a integridade física e psicológica, impondose a necessidade de um tratamento consistente (KOGA; FUREGATO, 2002; FELÍCIO; ALMEIDA, 2008). Portanto, para os profissionais entrevistados, a internação psiquiátrica torna-se essencial para reavaliar o caso clínico no intuito de restaurar ou desenvolver recursos e capacidades humanas essenciais, que se encontram desorganizadas no momento.

Diante disso, a saúde deve ser vista como um fenômeno integral e este nível só pode ser alcançado por um conjunto de especialidades em constante interação e diálogo. A necessidade e a busca por abordagens humanizadas às pessoas em sofrimento é cada vez maior por parte dos profissionais de saúde que as assistem, na tentativa de superação do enfoque biomédico e fragmentado aos indivíduos com transtorno mental (MORETTO, 2008; ALMEIDA, 2009; CORDEIRO et al., 2012).

$\mathrm{O}$ trabalho em equipe é bem evidenciado como prática diária dos profissionais entrevistados. A organização desse processo contempla a alta e média complexidade de saberes, bem como responsabilização coletiva das ações e a interação dos recursos humanos. Ao se trabalhar com a equipe multiprofissional ocorre um direcionamento para a reorganização do processo de trabalho nos serviços de saúde que visam modificações nas formas de agir sobre os fatores que compõem o processo de saúdedoença dos pacientes a partir de um maior entrosamento entre os profissionais e as ações que desenvolvem (COSTA; ENDERS; MENEZES, 2008; CORDEIRO et al., 2012).

Para acompanhar o processo de saúde em geral criou-se a Política Nacional de Humanização (PNH), de atenção e gestão no SUS, conhecida como Humaniza SUS; esse processo deve ser norteado pelos seguintes valores: autonomia, corresponsabilidade, protagonismo dos sujeitos envolvidos, solidariedade entre os vínculos estabelecidos, respeito aos direitos dos usuários e partici- 
pação coletiva no processo de gestão (MORETTO, 2008; CORDEIRO et al., 2012).

Para que ocorra a humanização na saúde, é necessário que se construam e se mantenham espaços de reflexão e de transformação constante das práticas profissionais. Um dos princípios que guiam essa política é o fortalecimento do trabalho em equipe multiprofissional e que, por meio de "decisões compartilhadas, a equipe possa se nutrir para novos desafios" (MORETTO, 2008, p. 27).

Essa prática de organização inovadora caracteriza-se pela necessidade de produção da atenção integral e humanizada aos pacientes, em correspondência ao conceito amplo de saúde e aos princípios do SUS. Essa atenção deve caracterizar-se pela ampliação dos referenciais profissionais e pelo reconhecimento da insuficiência da ação uniprofissional na resolução dos problemas de saúde dos pacientes (COSTA; ENDERS; MENEZES, 2008).

Os profissionais entrevistados reconheceram que as ações profissionais conjuntas e o compartilhamento de saberes irão destinar-se a cada paciente, levando em consideração o ser humano em sua singularidade. Diante disso, ressalta-se a importância da criação e da manutenção de um espaço no qual os profissionais estejam dispostos às trocas mútuas sobre as vivências de seu dia a dia profissional, para buscarem caminhos e soluções.

Por meio de reuniões realizadas como rotina na Instituição, os membros têm a preocupação de criar meios, que facilitem o desenvolvimento de possibilidades para perceberem possíveis lacunas nas condutas da equipe para, assim, criar condições para melhoria da qualidade de vida do paciente e da família (ABUHAB et al., 2005).

O trabalho em equipe, citado pelos entrevistados, além de possuir caráter enriquecedor em termo de intercâmbio de conhecimentos, visa também contribuir na rotina do paciente, através das especialidades que completam o quadro de profissionais na Instituição. Dessa maneira, as competências diversas de cada profissional serão integradas a partir de valores éticos, assegurando um espaço de interconexão entre os saberes e práticas. No projeto terapêutico, a preocupação maior deve ser a sua construção em uma dimensão participativa.

\section{CONCLUSÃO}

Por meio do presente estudo foi possível compreender a percepção e a vivência da equipe multiprofissional na assistência ao paciente esquizofrênico.

Para os profissionais, esta prática norteadora é executada trazendo enriquecimento e benefícios não somente para o processo de trabalho, mas também para o paciente que passa a ser considerado em toda sua complexidade e singularidade e não fragmentado, por especialidades, ou visto apenas pelo viés do transtorno mental.

O trabalho em equipe é fortalecido por meio da relação e laços estabelecidos entre os profissionais, que caminham juntos com o mesmo objetivo que é a estabilização desse paciente e melhorias na qualidade de vida.

A humanização apresenta-se tanto na relação com os pacientes, quanto entre os profissionais que buscam suporte entre si, para enfrentar as dificuldades e conhecer seus limites, em uma relação de autoajuda, pois reconhecem que no trabalho em saúde mental encontram-se diversos desafios, para os quais a cura efetiva, em determinadas situações, não é concebível.

Conclui-se, portanto, que o trabalho fragmentado torna-se uma prática incompatível com a atenção em saúde mental, necessitando da reorganização do processo de trabalho que visa a integração dos profissionais, bem como a interdisciplinaridade das especialidades. Essa comunicação e a humanização dos processos de cuidar devem considerar o paciente de maneira holística, como descrito nos depoimentos dos profissionais entrevistados.

\section{REFERÊNCIAS}

ABUHAB, D.; SANTO, A. B. A. P.; MESSENBERG, C. B.; FONSECA, R. M. G. S.; SILVA, A. L. A. O trabalho em equipe multiprofissional no CAPS III: um desafio. Rev Gaúcha Enferm., Porto Alegre, v. 26, n. 3; p. 369-380, 2005. Disponível em: < http://www.seer.ufrgs.br/RevistaGauchadeEnfermagem/article/view/4567/0> . Acesso em: 13 jul. 2013. 
ALMEIDA, M. M. Cuidadores de pacientes com esquizofrenia: a sobrecarga e a atenção em saúde. 2009. 54f. Dissertação (Mestre em Ciências na Saúde Coletiva) - Fundação Oswaldo Cruz, Belo Horizonte, BH, 2009.

ALMEIDA, M. M.; SCHALL, V. T.; MARTINS, A. M.; MODENA, C. M. Representações dos cuidadores sobre a atenção na esquizofrenia. PSICO, Porto Alegre, PUCRS, v. 41, n. 1, p. 110-117, 2010. Disponível em: < http://revistaseletronicas.pucrs.br/teo/ojs/index.php/revistapsico/article/ viewFile/5417/5223 > . Acesso em: 08 mar. 2013.

ARAÚJO, M. F. M.; LEMOS, A. C. S.; CARVALHO, C. M. L. O relacionamento terapêutico no cuidado dispensado a um esquizofrênico: narrativas de um diário de campo. Rev Bras Promoção da Saúde, Fortaleza, v. 20, n. 2, p. 116123, 2007.

BARDIN, L. Análise de conteúdo. Lisboa, Portugal: Edições 70, 2008.

BRASIL. Ministério da Saúde. Conselho Nacional de Saúde. Resolução 466 do CNS que trata de pesquisas em seres humanos e atualiza a resolução 196. Disponível em: < http://conselho.saude.gov.br/resolucoes/2012/ Reso466.pdf>. Acesso em: 17 jul. 2013.

CORDEIRO, F. R.; TERRA, M. G.; PIEXAK, D. R.; ELY, G. Z.; SILVA, A. A. Cuidados de enfermagem à pessoa com esquizofrenia: revisão integrativa. Rev Enferm UFSM, Santa Maria, v. 2, n. 1, p. 174-181, 2012. Disponível em: $<$ http://cascavel.ufsm.br/revistas/ojs-2.2.2/index.php/ reufsm/article/view/3123 > . Acesso em: 13 maio 2015.

COSTA, R. K. S.; ENDERS, B. C.; MENEZES, R. M. P. Trabalho em equipe de saúde: uma análise contextual. Ciência, Cuidado e Saúde, Maringá, v. 7, n. 4, p. 530-536, 2008.

FELÍCIO, J. L.; ALMEIDA, D. V. Abordagens terapêuticas às famílias na reabilitação de pacientes internados em hospitais psiquiátricos: relato de experiência. $\mathbf{O}$ Mundo da Saúde São Paulo, São Paulo, v. 32, n. 2, p. 249-253, 2008.

FERNANDES, M. M.; SAEKI, T. A vida social de pessoas com diagnósticos de esquizofrenia, usuárias de um centro de atenção psicossocial. Rev. Latino-Am. Enfermagem, São Paulo, v. 17, n. 6, p. 995-1000, 2009.
KOGA, M.; FUREGATO, A. R. Convivência com a pessoa esquizofrênica: sobrecarga familiar. Ciência, Cuidado e Saúde, Maringá, v. 1, n. 1, p. 69-73. 2002. Disponível em: <http://www.periodicos.uem.br/ojs/index.php/ CiencCuidSaude/article/view/5656 > . Acesso em: 13 maio 2015.

LAUTER, D. S.; DALLABRIDA, F. A.; SILVEIRA, C. O.; CASAROTTO, M. E.; KOLANKIEWICZ, A. C. B. Estudo de caso de um paciente com diagnóstico de esquizofrenia e depressão. Rev Contexto e Saúde, Ijuí, v. 10, n. 20, p. 957-962, 2011. Disponível em: < https://www.revistas. unijui.edu.br/index.php/contextoesaude/article/viewFile/1707/1414_> . Acesso em: 15 maio 2015.

LIMA, D. U.; GARCIA, A. P. R. F.; TOLEDO, V. P. Olhares da enfermagem para o paciente esquizofrênico: implicações do processo saúde doença para o cuidado. Rev Enferm UFPE, Recife, v. 7, n. 5, p. 4325-4331, 2013.

MORETTO, C. C. Experiências de uma equipe interdisciplinar de saúde mental: um estudo psicanalítico. 2008. 123f. Dissertação (Mestre em Psicologia como Profissão e Ciência) - Pontifícia Universidade Católica, Campinas, 2008.

NOTO, C. S.; BRESSAN, R. A. Esquizofrenia: avanços no tratamento multidisciplinar. 2. ed. Porto Alegre: Artmed, 2012.

ORGANIZAÇÃO MUNDIAL DE SAÚDE. OMS. Organização Pan-Americana de Saúde. Relatório sobre a Saúde no mundo 2001. Saúde Mental: nova concepção, nova esperança. Genebra: OMS, 2001.

RESENDE, A. C.; VIGLIONE, D. J.; ARGIMON, I. I. L. Diferenças cognitivas entre os gêneros na esquizofrenia através do Rorschach. PSICO, Porto Alegre, PUCRS, v. 40, n. 3, p. 279-286, 2009.

Recebido em: 03 novembro 2015 Revisado em: 24 janeiro 2016 Aceito em: 23 fevereiro 2016 